\title{
Obesity and non-insulin-dependent diabetes mellitus in Swiss-Webster mice associated with late-onset hepatocellular carcinoma
}

\author{
Laura B Lemke ${ }^{1}$, Arlin B Rogers ${ }^{1}$, Prashant R Nambiar ${ }^{1}$ and James G Fox ${ }^{1,2}$ \\ ${ }^{1}$ Division of Comparative Medicine and ${ }^{2}$ Department of Biological Engineering, Massachusetts Institute of Technology, Building 16-820, Cambridge, \\ Massachusetts 02139, USA \\ (Correspondence should be addressed to L B Lemke at Animal Resources Center, University of Texas at Austin, 1 University Station, A2500, Austin, TX \\ 78712-0136, USA; Email: Ilemke@mail.utexas.edu)
}

\begin{abstract}
Genetic mutations resulting in obesity and type 2 diabetes mellitus (T2D) are described for both inbred and outbred mice. However, no known mouse model completely recapitulates human $\mathrm{T} 2 \mathrm{D}$ and its comorbidities. We identified a cohort of obese, male, outbred Swiss-Webster (SW) mice as polyuric, polydipsic, glucosuric, and hyperglycemic. Prevalence of glucosuria in the SW colony reached $60 \%(n=70)$ in males 8 weeks to 6 months of age. Despite severe obesity in some females, no females were diabetic. Pathologic findings in affected males included cachexia, dilated gastrointestinal tracts with poor muscular tone, pancreatic islet degeneration and atrophy with compensatory metaplasia and/or neogenesis, bacterial pyelonephritis, membranous glomerulopathy, and late-onset hepatic tumors with macrosteatosis, microsteatosis, and hydropic change in aged males. Serum insulin
\end{abstract}

correlated with blood glucose in a nonlinear pattern, suggestive of islet exhaustion. Circulating leptin levels showed a weak inverse correlation with glucose. Diabetic males were bred with obese colony females to produce 20 male and 20 female offspring. Prevalence of diabetes in male offspring was $80 \%(16 / 20)$ with a median age of onset of 18 weeks. By contrast, no diabetic females were identified, despite being significantly more obese than males. Male predominance is likewise a feature of T2D in humans. To our knowledge, this is the first documentation of hepatocellular carcinoma and islet metaplasia and/or neogenesis in a spontaneous outbred mouse model of T2D. The SW availability and histopathologic features represent a promising new model for the study of T2D.

Journal of Endocrinology (2008) 199, 21-32

\section{Introduction}

The incidence of obesity and its comorbidities - most notably metabolic syndrome, type 2 diabetes (T2D) and cardiovascular disease (CVD) - continues to increase in both adults and adolescents (Rosenbaum et al. 2004). Metabolic syndrome is defined by the presence of at least three of the following risk factors: abdominal obesity, dyslipidemia, hypertension, insulin resistance, a proinflammatory state (elevated C-reactive protein), and a prothrombotic state (elevated plasma plasminogen activator inhibitor; Grundy et al. 2004). A majority of metabolic syndrome patients are obese and insulin resistant, conferring an increased risk for T2D (Grundy et al. 2004). Humans with T2D have a functional insulin deficiency characterized by fasting hyperglycemia due to peripheral insulin resistance combined with an insulin secretory defect (i.e., inability to increase $\beta$-cell mass).

An estimated $5-10 \%$ of the US population suffers from T2D (Gregg et al. 2004, Sullivan et al. 2005) and is at increased risk for developing microvascular and neuropathic complications, macrovascular disease, microbial infections, and neoplasia. Recently, several epidemiologic studies have shown an increased risk of hepatocellular carcinoma (HCC) in patients with T2D in the absence of viral hepatitis (El-Serag 2004, Davila et al. 2005). HCC in humans with diabetes is associated with underlying nonalcoholic fatty liver disease (NAFLD), a common finding in patients with obesity and metabolic syndrome. NAFLD can progress to steatohepatitis with cirrhosis and eventual neoplastic transformation. While the direct link between diabetes and HCC remains unknown, underlying chronic liver disease has been implicated in the development of both diabetes and HCC.

Several spontaneous and induced genetic mutations resulting in varying degrees of obesity and diabetes have been described in mice, making them popular models in diabetes research (Accili 1995, Rees \& Alcolado 2005). Mutation of the leptin or leptin receptor gene produces obesity and T2D in the ob/ob and db/db mouse respectively. The $\mathrm{db} / \mathrm{db}$ mutation is available on a variety of mouse strain backgrounds, is well characterized, and displays many of the comorbidities of T2D found in humans, including CVD, renal disease, and neuropathies (Robertson \& Sima 1980, Sharma et al. 2003, Ye et al. 2005). However, these mice represent a monogenic model demonstrating features related to leptin resistance, including morbid obesity, hyperphagia, and 
alterations in thermoregulation and fertility. In contrast to popular mouse models of diabetes, common forms of human T2D are considered polygenic disorders, occurring through the simultaneous action of several genes and rarely involving alterations of the leptin/leptin receptor axis (McCarthy \& Menzel 2001).

We recently identified a cohort of male, outbred SwissWebster (SW) mice in a breeding colony that required frequent cage changes due to urine-soaked bedding and frequent water bottle changes (i.e., polyuria/polydipsia). The SW breeding colony at the Massachusetts Institute of Technology (MIT) is maintained to provide mice for the embryo transfer program and occasionally for sentinel animals as part of the rodent health monitoring program. At the time of the study, the colony had been closed for $\sim 2$ years with a random breeding strategy in place. Five males were isolated from the colony for further study. Metabolic comparisons with age-matched, control males showed markedly increased urine production and water consumption in the affected mice; however, there were no significant differences in food consumption or body weight (data not shown). Additionally, affected mice were glucosuric, and serum chemistry analysis with insulin levels on one mouse revealed hyperglycemia, hypercholesterolemia, azotemia, and hyperinsulinemia - signs consistent with both metabolic syndrome and T2D in humans. The affected mice were subsequently bred with females from the SW colony to produce 20 male and 20 female offspring. The present study describes the clinicopathologic features of T2D in these SW offspring as well as the prevalence and characterization of this disease in the SW breeding colony where the disease was first recognized.

\section{Materials and Methods}

\section{Animal sources and husbandry}

Five, 18-week-old, male, outbred SW mice and ten, 8-weekold, female SW mice were obtained from the breeding stock of the MIT Division of Comparative Medicine mouse breeding program (closed breeding program for a minimum of 2 years), and subsequently bred to produce 20 male and 20 female offspring (F1's). SW offspring were weaned at 4 weeks of age and separated by sex into groups of four. These mice have continued to be inbred, and mice up to generation 7 are included in the aging part of this study. Additional diabetic and nondiabetic male and nondiabetic female SW mice were selected from existing sentinel mouse cages placed in mouse rooms on the MIT campus.

Mice were housed in polycarbonate microisolator cages on hardwood bedding (PharmaServ, Framingham, MA, USA) under specific pathogen-free conditions (free of Helicobacter spp., Citrobacter rodentium, Salmonella spp., endoparasites, ectoparasites, and known murine viral pathogens) in an Association for the Assessment and Accreditation of Laboratory Animal Care International accredited facility.
Mouse rooms were kept at constant temperature and humidity on a $12 \mathrm{~h}$ light: $12 \mathrm{~h}$ darkness cycle, and mice were provided with standard rodent chow (Purina Mills, St Louis, MO, USA) and water ad libitum. All protocols were reviewed and approved by the MIT Committee on Animal Care. Mice were killed by $\mathrm{CO}_{2}$ inhalation in accordance with the guidelines set by the American Veterinary Medical Association (Beaver et al. 2001).

\section{Clinicopathology of colony SWs}

The presence of glucosuria was determined by collection of urine from non-anesthetized mice onto glucose urine dipsticks, according to the manufacturer's instructions (Diastix; Bayer Corporation). Based on the presence or absence of glucosuria, mice were defined as diabetic or nondiabetic.

Fifteen diabetic male colony SW mice and five nondiabetic colony SW mice (three males, two females), $\sim 6$ months of age, were selected for necropsy. Non-fasting blood glucose concentrations were measured from $10 / 15$ glucosuric and $5 / 5$ non-glucosuric mice using the One Touch Basic glucometer (LifeScan Inc., Milpitas, CA, USA) from whole blood collected via terminal cardiac puncture. Whole blood was analyzed for complete blood count and serum was submitted to a reference laboratory (IDEXX, North Grafton, MA, USA) for biochemical analysis, insulin concentration, and insulin-to-glucose ratio. Urine was collected via cystocentesis and submitted for complete urinalysis and aerobic culture.

\section{Breeding experiments}

To assess the reproductive performance of diabetic mice, five 6-month-old, diabetic male SW mice and ten nondiabetic females were selected from the breeding colony and bred to produce 20 male and 20 female F1 offspring.

Selected F1 brother-sister matings were undertaken to gauge reproductive viability and to establish diabetic SW (dSW) and nondiabetic SW (ndSW) lines. For diabetic mice, males were selected for breeding at the first positive test for glucosuria and mated with the heaviest female from the same litter. In nondiabetic mice, the smallest male and female were selected for breeding. Subsequent litters were inbred using the same paradigm, with mice up to generation 7 included in this study.

\section{Pathology and immunohistochemistry (IHC)}

Tissues were collected at scheduled necropsy from diabetic and nondiabetic parent colony SW, as well as selectively bred dSW and ndSW lines. For routine histopathology, the tissues were fixed in 10\% neutral buffered formalin, routinely processed and embedded, sectioned at $4 \mu \mathrm{m}$, and stained with hematoxylin and eosin $(\mathrm{H} \& \mathrm{E})$. Formalin-fixed, whole pancreata were sectioned at $4 \mu \mathrm{m}$ to include the gastric (head) and duodenal (tail) portions for immunohistochemical staining using guinea pig anti-swine insulin antibody (30) (DakoCytomation, 
Carpinteria, CA, USA) at 1:150 on an automated immunostainer as described previously (Riu et al. 2002).

\section{Bacteriology}

Urine collected via cystocentesis, and aseptically collected tissue samples from gross lesions of the kidney, urinary bladder, liver, and/or gallbladder were submitted in trypticase soy broth for aerobic culture at $37^{\circ} \mathrm{C}$ when appropriate.

\section{Establishment and characterization of $d S W / n d S W$}

dSW offspring produced during breeding experiments with the diabetic colony SW males were characterized during development to determine the onset of clinical signs of obesity and diabetes. Mice were weaned at 4 weeks of age and separated by gender into ten groups of four mice/group to total 20 males and 20 females (at least four males and four females were included from each fertile sire). At weaning, body weight was recorded and mice were tested for the presence of glucosuria using the urine dipsticks. Urine glucose was reassessed at $10,18,26$, and 30 weeks to determine the median age of onset of glucosuria. Body weight was recorded at 1 -week intervals until week 9 and then at weeks 17, 19, 22, 26, and 30. Blood was collected at 13 and 30 weeks via the retro-orbital sinus, from non-fasted mice anesthetized with $2 \%$ isoflurane in oxygen for the determination of blood glucose using the One Touch Basic glucometer (LifeScan Inc). Serum insulin and leptin concentrations were measured from the 30-week serum samples using rat/mouse insulin and mouse leptin ELISA kits respectively (Linco Research, St Charles, MO, USA), according to the manufacturer's instructions.

Glucose tolerance testing was performed in selected diabetic and nondiabetic male and female mice. After a 12- to 14-h fasting period, baseline blood glucose measurements were obtained using whole blood collected from the tip of the tail in unanesthetized mice. Then, $1.5 \mathrm{mg}$ glucose per gram body weight was administered via i.p. injection $(75 \mathrm{mg} / \mathrm{ml}$ solution) and serial blood samples were obtained and analyzed at $15 \mathrm{~min}$, $30 \mathrm{~min}, 60 \mathrm{~min}, 90 \mathrm{~min}$, and $120 \mathrm{~min}$ post-injection.

\section{Statistical methods}

Body weights and serum chemistries were analyzed by oneway ANOVA with Tukey's post hoc test. Median survival was calculated by Kaplan-Meier survival curve analysis. Serum insulin and leptin concentrations were compared with blood glucose concentration by nonlinear and linear regression analysis respectively. Glucose tolerance testing was analyzed by the determination of the areas under the curve (AUC). Mean AUCs for diabetic and nondiabetic groups were compared using an unpaired t-test. $P<0.05$ were considered significant. All analyses utilized GraphPad Prism version 4.0 for Windows (GraphPad Software, San Diego, CA, USA).

\section{Results}

\section{Characterization of diabetes in $d S W$ mice}

Prevalence of diabetes in the male dSWs was $80 \%(n=20)$ with a median age of onset of 18 weeks (Fig. 1A), and no diabetic females were identified. Mean, non-fasting blood glucose of diabetic male offspring at 30 weeks was $451 \cdot 3 \pm$ $93.9 \mathrm{mg} / \mathrm{dl}$ and differed significantly from nondiabetic male and female offspring $(163 \cdot 7 \pm 11 \cdot 3$ and $171 \cdot 3 \pm 25 \cdot 2 \mathrm{mg} / \mathrm{dl}$ respectively, $P<0 \cdot 01$; Fig. 1B). Female offspring were significantly heavier than males at $53.9 \pm 5.7 \mathrm{~g}$ and $48 \cdot 2 \pm$ $6 \cdot 0 \mathrm{~g}$ respectively $(P<0 \cdot 05)$ at 30 weeks of age (Fig. 2$)$.

Serum insulin concentrations for dSWs were measured using a rat/mouse insulin ELISA kit (Lincoplex). Serum insulin concentration was highly variable between mice, with no significant differences observed between the mean values for diabetic (males) and nondiabetic (male and female) mice. However, insulin concentrations showed a significant, nonlinear correlation with blood glucose concentrations in $\mathrm{dSW}$ males at 7-9 months of age (Fig. 3). Insulin concentrations were increased in $\mathrm{dSW}$ with borderline and mildly elevated blood glucose concentrations and were the highest in those mice with a blood glucose concentration of $\sim 300 \mathrm{mg} / \mathrm{dl}$ (coincident with an onset of overt glucosuria). Diabetic mice with blood glucose concentrations above $300 \mathrm{mg} / \mathrm{dl}$ showed declining insulin concentrations that were inversely proportional to blood glucose concentration.

Glucose tolerance testing in fasted mice revealed elevated glucose curves in response to glucose challenge in diabetic male mice compared with nondiabetic mice. Mean AUC for diabetic mice was significantly higher than nondiabetic mice (25 420 \pm 1501 and $13060 \pm 992$ respectively, $P<0 \cdot 0005$; Fig. 4), consistent with glucose intolerance in dSW males. Glucose tolerance curves were variable between mice as blood glucose measurements in mice over 12 months of age were difficult to interpret due to the high prevalence of liver tumors in diabetic males. The presence of liver tumors occasionally coincided with a reversion to euglycemia or hypoglycemia in some mice with previously noted hyperglycemia.

Serum leptin concentrations, measured by a mouse leptin ELISA kit (Lincoplex), were also highly variable between mice, and no significant differences in mean concentrations were observed between diabetic and nondiabetic mice $(6 \cdot 821 \pm 6.920$ and $8.766 \pm 4.385 \mathrm{ng} / \mathrm{ml}$ respectively). However, serum leptin concentration showed a significant, inverse linear correlation with blood glucose concentration in diabetic 7-month-old dSW males (Fig. 5).

Diabetic dSW male and female mice produced viable offspring (average 12 pups) when bred at less than 6 months of age and prior to the onset of moderate obesity in the females $(<50 \mathrm{~g})$. No females became pregnant when bred after 6 months of age $(n=3)$; however, dSW males remained reproductively viable past 8 months of age ( $8 / 8$ tested males were capable of impregnating females and producing offspring). 

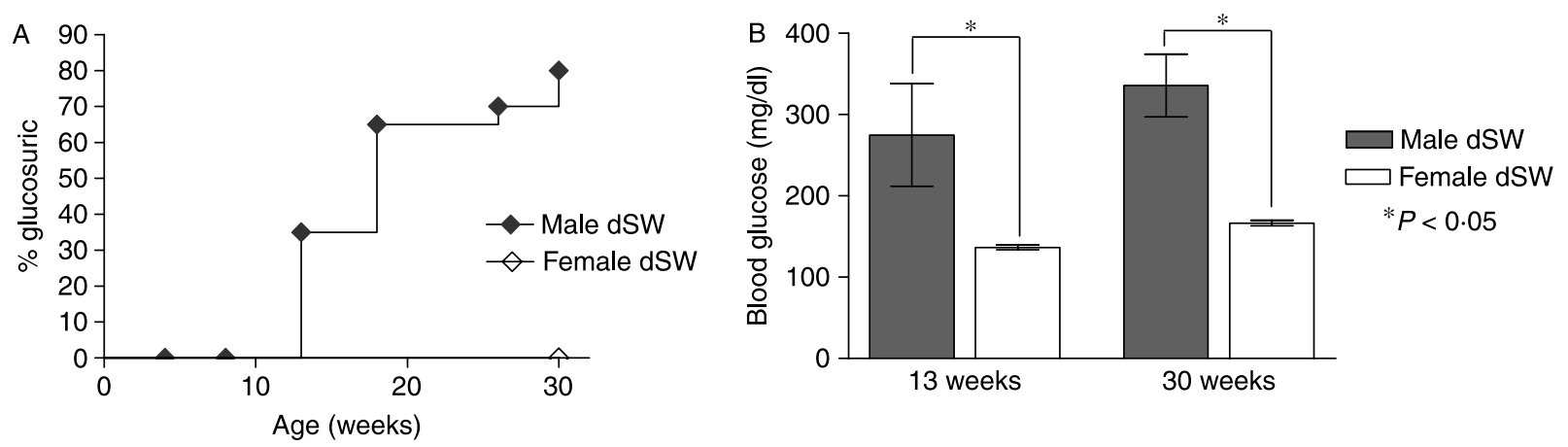

Figure 1 Characterization of diabetes in male and female dSWs. (A) Onset of glucosuria in male dSWs. Kaplan-Meier survival curve where onset of glucosuria was considered as event. Median survival in male mice $(n=20)$ was 18 weeks of age. Throughout the test period, no glucosuric females $(n=20)$ were identified. (B) Hyperglycemia in male dSWs. Non-fasting, whole blood glucose

concentration was significantly higher in male dSWs $(n=20)$ compared with in age-matched dSW females $(n=20)$ at both 13 and 30 weeks of age as measured using a bedside glucometer. Blood glucose concentration was more variable in male mice compared with females with a greater proportion of males showing significant hyperglycemia at 30 weeks of age compared with 13 weeks. Mean values with standard error margins are displayed.

\section{Diabetic parent colony SW mice}

Over a sampling period of 1 week, the prevalence of diabetes (defined as blood glucose $>300 \mathrm{mg} / \mathrm{dl}$ with glucosuria) in the SW mouse breeding colony was 60\% (42/70) in males between 8 weeks and 12 months of age (average age $>6$ months). The prevalence of diabetes in male SW housed in a corridor separate from the breeding colony was 36\% (17/47). However, the average age of males housed in the second corridor was $<4$ months and no breeding animals were tested. No females from either colony tested positive for glucosuria $(n=40)$.

Serum biochemical data were analyzed in nine dSW and three ndSW males, $\sim 6$ months of age. The mean blood glucose concentration in diabetic (i.e., glucosuric) males was significantly higher than nondiabetic males at $396 \cdot 2 \pm 14 \cdot 4 \mathrm{mg} / \mathrm{dl}$

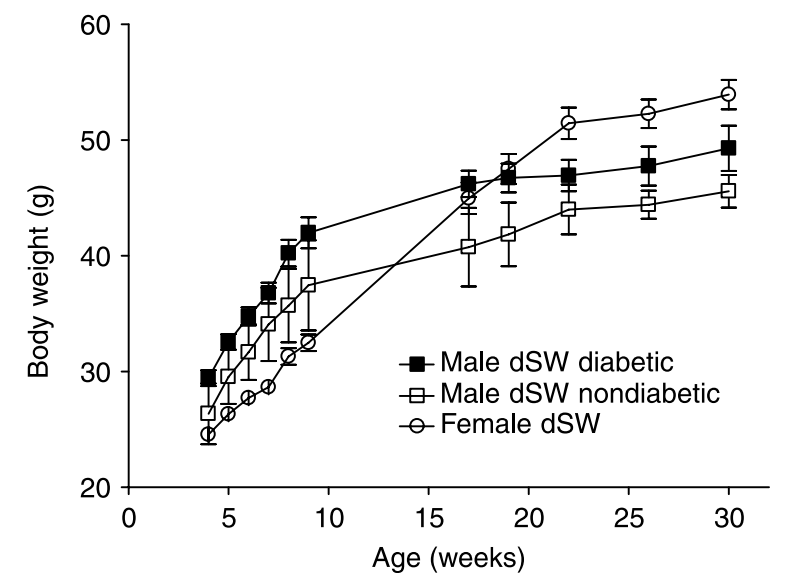

Figure 2 Growth rate in dSWs. Body weight was measured over time in male $(n=20)$ and female $(n=20)$ dSW mice. At 30 weeks of age, diabetic male dSW were significantly heavier than nondiabetic male dSW. Although weanling males were initially heavier than females, at 30 weeks of age, females were significantly heavier than males. Mean values with standard error margin are displayed. and $235 \cdot 3 \pm 35 \cdot 59 \mathrm{mg} / \mathrm{dl}$ respectively $(P=0 \cdot 0005)$. Diabetic males had significant elevations in serum alanine aminotransferase, blood urea nitrogen, phosphorus, and lipemic index compared with nondiabetic males (Fig. 6). These alterations were consistent with early pre-renal or renal insufficiency, hepatocellular injury, and hyperlipidemia.

\section{Gross pathology}

Gross and histologic lesions in diabetic male mice, whether from the parent colony or dSW line, were similar in character. Diabetic male mice with advanced disease (blood glucose $>300 \mathrm{mg} / \mathrm{dl}$, urine glucose positive) were distinguishable grossly from nondiabetic mice based on decreasing body condition. External gross findings included urine staining of the perineum and ventrum, unkempt hair coats, abdominal distension, and decreased body condition scores (UllmanCullere \& Foltz 1999). Body condition scores averaged 2 out of 5 as ascertained by palpation of the iliac bones and sacral

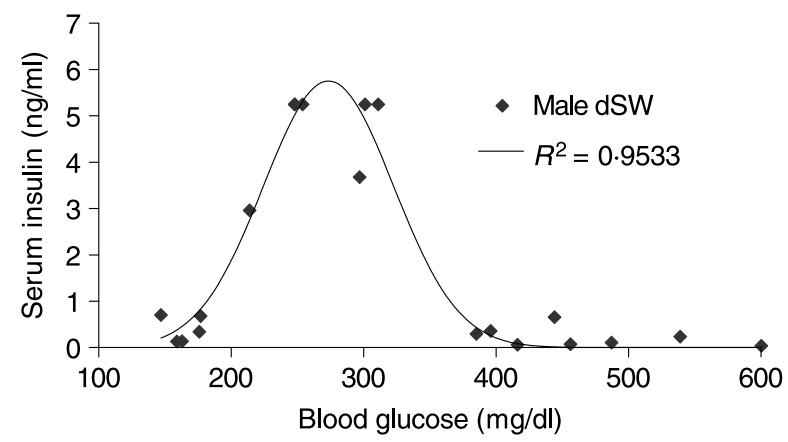

Figure 3 Insulin and blood glucose concentrations in male dSWs $(n=20)$ at 7 months of age. Non-fasting serum insulin measured by ELISA showed a nonlinear correlation with whole blood glucose concentration. Male dSW with mild hyperglycemia showed elevated insulin concentrations compared with males with marked hyperglycemia. 

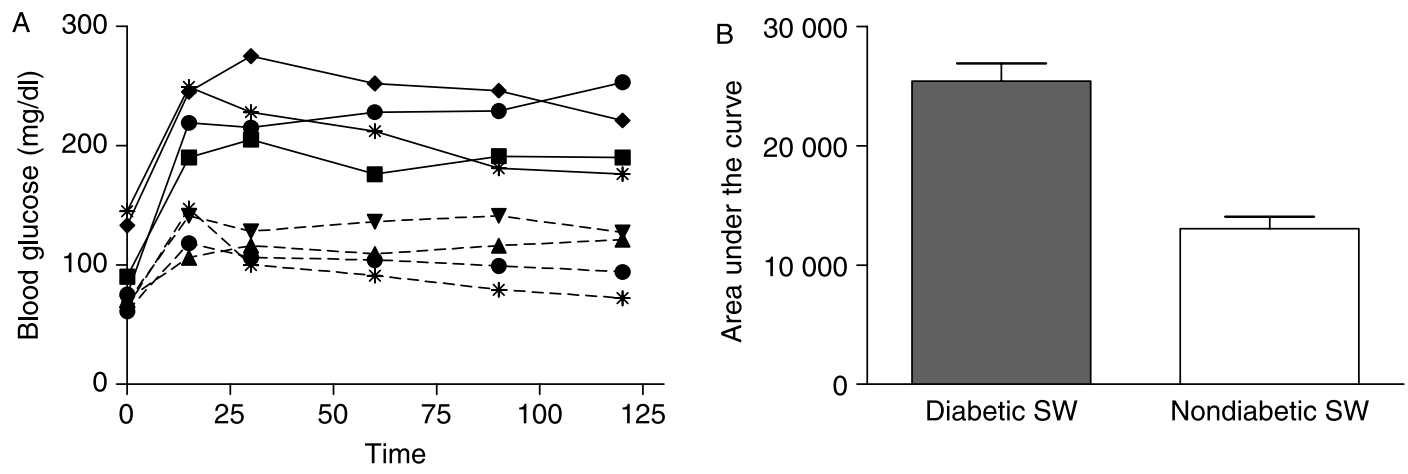

Figure 4 Glucose tolerance testing in aged dSW and ndSW at 12 months of age. (A) Glucose tolerance curves revealed elevated baseline with delayed return to baseline in diabetic males (solid lines) compared with nondiabetic males (broken lines). (B) AUC measurements obtained from the curves in (A) were significantly higher in diabetic compared with nondiabetic males.

prominences. Despite poor body conditions, diabetic mice maintained body weight due to abdominal distension (Fig. 7) caused by enlarged, flaccid, ingesta-filled gastrointestinal tracts. The abdominal musculature of diabetic mice was thin and transparent, and the cecum markedly dilated and filled with ingesta, when compared with age- and gendermatched, nondiabetic controls (Fig. 7). Dilation of the stomach and small intestine was evident less frequently. Diabetic males in the initial stages of disease showed increased body weight and abdominal fat deposition compared with nondiabetic males.

Kidneys of mice with ascending urinary tract infections were enlarged and irregularly disfigured by pelvic and, less frequently, cortical pale-to-yellow caseous abscesses up to $5 \mathrm{~mm}$ in diameter. Mice with marked pyelonephritis became moribund, exhibiting hunched posture, reluctance to move, poor body condition, and an unkempt appearance. Ascending urinary tract infections were a significant cause of morbidity and mortality in the diabetic cohort. One mouse had uroabdomen and was suspected to have ruptured the ureter near the renal pelvis. Diabetic mice also showed mildly to moderately enlarged kidneys bilaterally with mild dilation of the renal pelvises and ureters (hydronephrosis and hydroureter).

Diabetic mouse livers appeared moderately enlarged and pale compared with nondiabetic mouse livers. Seventy-five percent of diabetic males, $12-18$ months of age $(n=12)$, had grossly visible hepatic tumors. Liver tumors were not observed in age-matched nondiabetic males $(n=4)$ or females $(n=5$; odds ratio $=24 \cdot 43, P=0 \cdot 008)$. Tumors ranged from $0 \cdot 1 \times 0.1 \mathrm{~cm}$ to $1.5 \times 2.0 \mathrm{~cm}$. Affected mice frequently exhibited multiple tumors affecting multiple liver lobes, with only 3 out of 12 affected mice showing a single tumor. Interestingly, obese females but not males from the parent
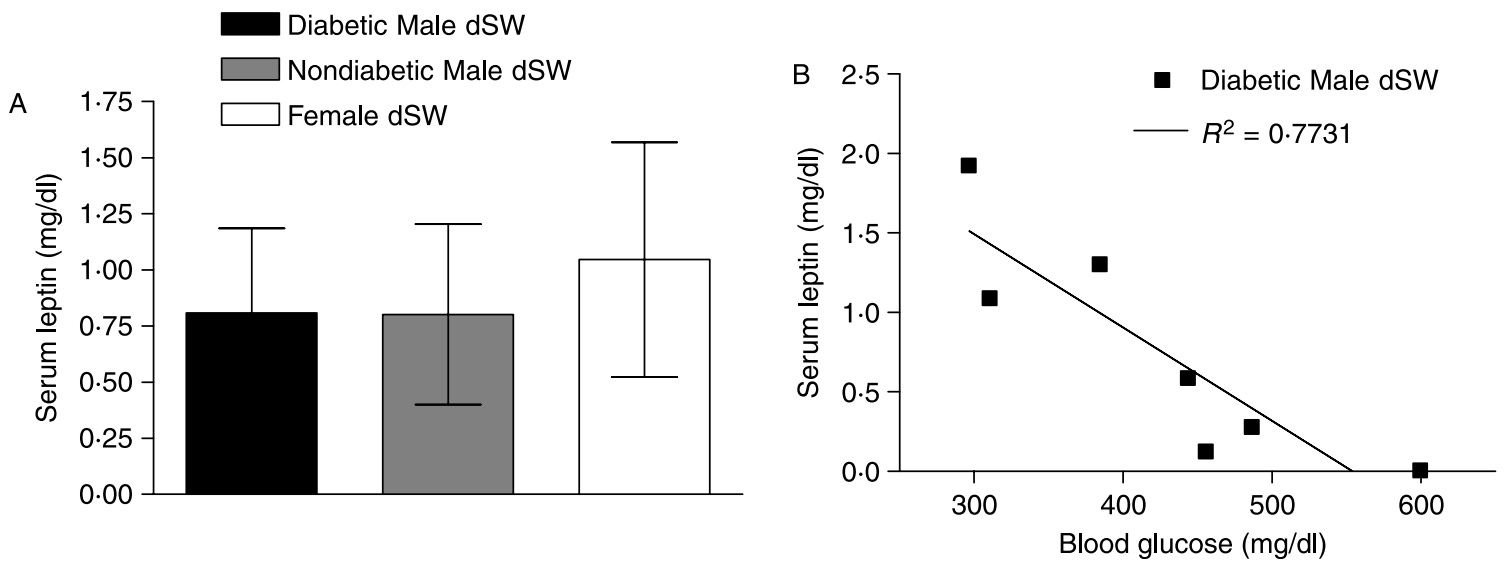

Figure 5 Serum leptin in dSW at 7 months of age. Non-fasting serum leptin concentrations as measured by ELISA were highly variable among diabetic male $(n=17)$, nondiabetic male $(n=7)$, and female $(n=4) \mathrm{dSW}$ with no significant differences observed. In diabetic male dSW, leptin concentrations showed a weak inverse correlation with whole blood glucose concentration. Markedly hyperglycemic males also showed decreased adipose stores and body condition, which may explain decreased leptin concentrations. 

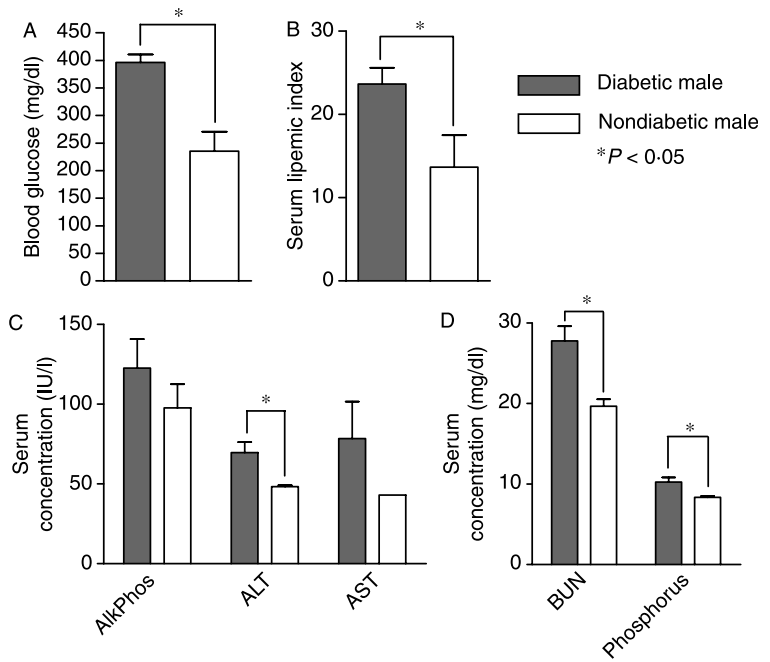

Figure 6 Biochemical characterization of diabetes in colony SW males. Diabetic male SW $(n=9)$ mice showed significantly higher (A) non-fasting, whole blood glucose concentration (B) and serum lipemic index, when compared with nondiabetic male SW $(n=3)$. Serum alanine aminotransferase (ALT) and blood urea nitrogen (BUN) were also significantly elevated ( $C$ and $D$ respectively) in diabetic male SW, but not serum creatinine (data not shown), suggesting pre-renal or early renal azotemia. Mean values with standard error margin are displayed.

colony infrequently developed non-obstructive pigmented gallstones as determined by microscopic morphology and polarization pattern.

\section{Histopathology}

The pancreata of diabetic and nondiabetic mice were scored from 0 (none) to 4 (severe) for islet haphazard arrangement and vacuolation, and 0 (focal) to 4 (severe) for histologic changes consistent with islet metaplasia (Fig. 8). Virtually all male mice, whether clinically diabetic or not, displayed some degree of islet cell pleomorphism and atypia. Enlarged islets and mega-islets were occasionally seen in diabetic male mice (Fig. 9). Islet metaplasia was defined by incomplete de novo parenchymal sublobulation, well removed from ductular regions, with a mixture of cells displaying islet, acinar, and/ or intermediate morphology (Fig. 9). Because of the clinical history and characteristic histologic pattern, these regions were interpreted as zones of exocrine pancreatic acinar cells undergoing metaplasia to an islet phenotype. In other instances, cells with an islet phenotype were observed in small, poorly defined clusters adjacent to ducts. These foci were defined as islet neogenesis based on immature morphology and location near the normal site of islet development. In some mice, radiating cords of islet-type cells emanated from periductular regions and extended haphazardly into surrounding acinar regions (Fig. 9). In many diabetic male mice, pancreatic ducts were ectatic and filled with secretory material, suggesting hypersecretion
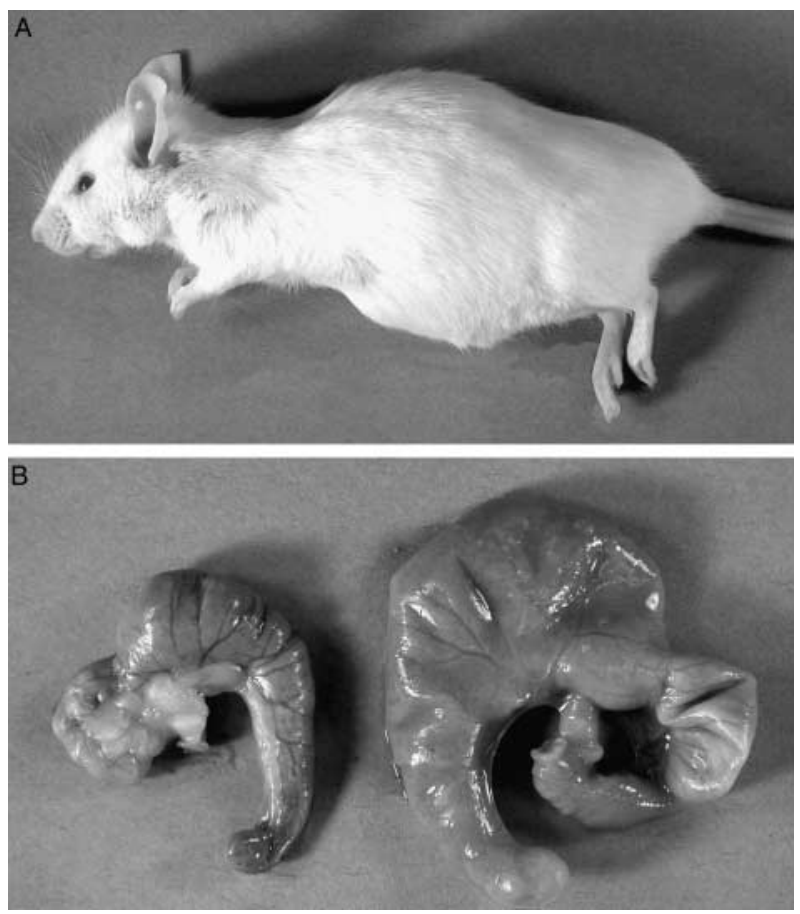

Figure 7 Gross pathology of diabetic colony SW males. (A) Diabetic male colony SW mice with advanced disease were grossly distinguishable from nondiabetic males, showing decreased body condition scores, unkempt fur and a pot-bellied appearance due to enlarged, ingesta-filled gastrointestinal tracts. (B) The ceca of diabetic males (right) were enlarged and flaccid compared with age-matched nondiabetic males (left). Gross findings suggested decreased gastrointestinal motility.

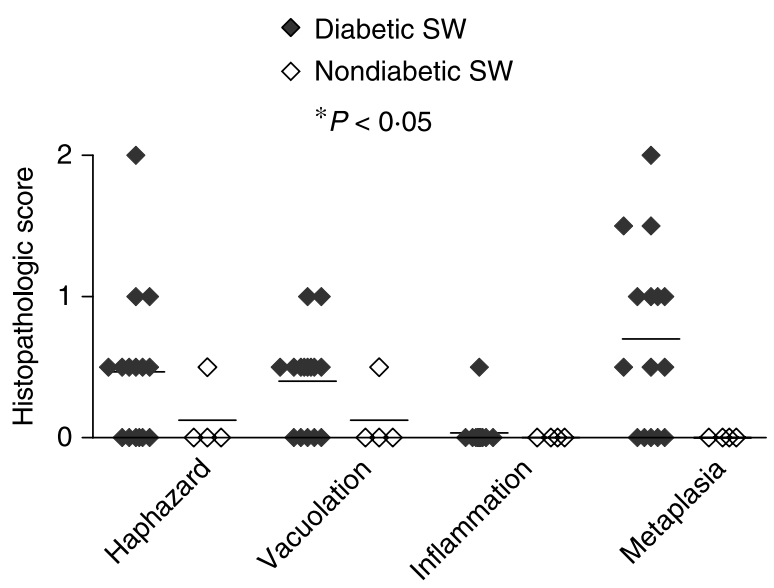

Figure 8 Pancreatic pathology scores in diabetic $(n=15)$ and nondiabetic $(n=4)$ colony SW mice. The pancreata of diabetic and nondiabetic mice were scored from 0 (none) to 4 (severe) for islet haphazard arrangement, vacuolation, inflammation, and changes consistent with islet metaplasia. Islet metaplasia was only observed in diabetic males. 

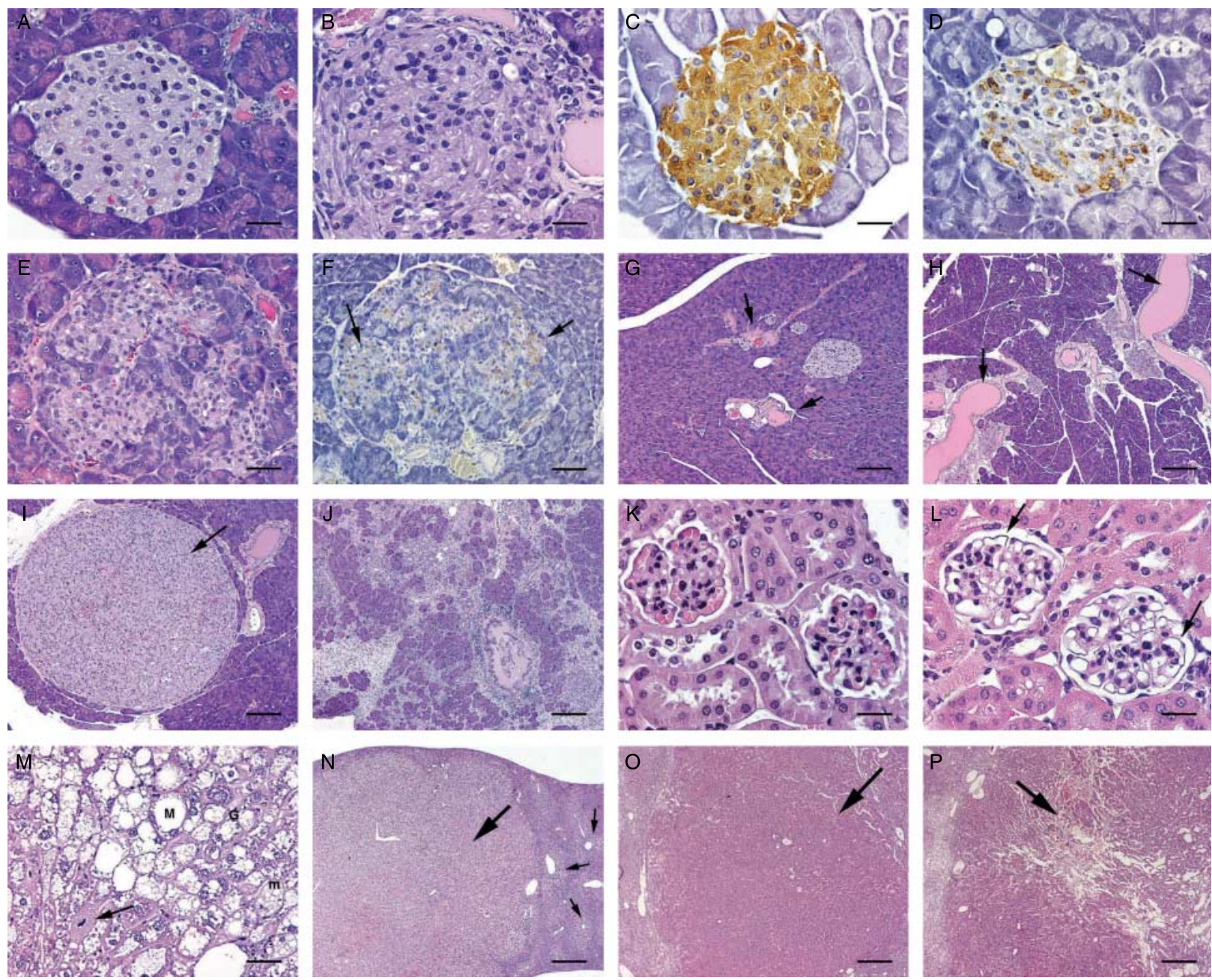

Figure 9 Histopathologic and immunohistochemical features of SW T2D. (A) Normal pancreatic island from obese but nondiabetic female mouse. (B) Islet from diabetic male demonstrating irregular cell arrangement and variable cytoplasmic swelling by granular to fibrillar pale eosinophilic material. (C) Insulin immunohistochemistry (IHC) demonstrating uniform distribution of insulin in female pancreatic islet. (D) Insulin IHC of diabetic male pancreatic islet shows patchy punctate staining in a subset of cells. (E) Islet metaplasia characterized by newly encapsulated sublobule containing a mixture of exocrine and endocrine pancreatic cell phenotypes. (F) Insulin IHC of islet metaplasia highlights irregular expression limited to endocrine cell phenotypes (arrows). (G) Female pancreas demonstrating normal ducts (arrows). (H) Diabetic male pancreas with ectatic ducts filled with secretory material (arrows) suggesting hypersecretion and/or outflow impairment. (I) Mega-islet in diabetic male. (J) Sclerosing pancreatitis in diabetic male characterized by replacement of acinar cells with wide bands of fibrous connective tissue, and mixed inflammatory cells surrounding ducts and infiltrating septa. (K) Normal glomeruli from female kidney. (L) Membranous glomerulopathy of kidney in diabetic male characterized by decreased erythrocyte profiles and thickened endothelial basement membranes (wire-loop capillaries; arrows). (M) Liver of aged ( $>1$ year) diabetic male demonstrating mixed fatty and hydropic hepatocyte degeneration with large lipid vacuoles ( $M$, macrosteatosis), small lipid vacuoles $(\mathrm{m}$, microsteatosis), and cloudy swelling characteristic of glycogen accumulation $(\mathrm{G})$; adjacent hepatocytes had an increased proliferative index (mitotic figure at arrow). (N) Clear cell hepatocellular carcinoma (HCC; large arrow) arising from liver lobe of aged male mouse with preexisting centrilobular fatty and hydropic degeneration (small arrows). (O) Solid nodule (arrow) within larger mixed phenotype HCC. (P) Trabecular and pelioid tumor differentiation (arrow) within mixed HCC. (A, B, E, G-P) Hematoxylin and eosin, and (C, D and F) insulin IHC; bar $=25 \mu \mathrm{m}(\mathrm{A}-\mathrm{D}, \mathrm{K}$ and $\mathrm{L}), 50 \mu \mathrm{m}(\mathrm{E}, \mathrm{F}$ and $\mathrm{M}), 250 \mu \mathrm{m}(\mathrm{G}-\mathrm{J}), 500 \mu \mathrm{m}(\mathrm{N}-\mathrm{P})$.

and/or outflow obstruction. Leakage from ectatic ducts may have resulted in a sclerosing pancreatitis observed in one aged, diabetic male (Fig. 9). Although remarkable in presentation and variety, pancreatic islet histopathology did not correlate with glucosuria nor serum glucose or insulin levels in mice for which data were available. No significant pancreatic lesions were observed in female mice regardless of body weight or blood glucose level.

Diabetic mice were at increased risk for renal disease including increased numbers of mesangial cells, mesangial 
matrix expansion, and dilated renal pelvises, as listed in Table 1. Kidney lesions in diabetic mice were more severe with advancing age, displaying membranous glomerulopathy characterized by decreased erythrocyte profiles and thickened endothelial basement membranes (Fig. 9). A portion of diabetic mice developed ascending pyelonephritis as evidenced by kidney abscessation, pyuria, and bacteriuria. Intralesional bacterial colonies were readily apparent by both H\&E and tissue Gram's stain.

Livers of aged ( $>1$ year) diabetic males $(n=12)$ and less frequently in mice less than 1 year of age exhibited moderateto-severe centrilobular glycogen-associated hydropic degeneration superimposed over microvesicular and macrovesicular steatosis, and several had HCC. Steatosis was centrilobular in distribution and adjacent mid-zonal hepatocytes frequently demonstrated cellular atypia and an increased mitotic rate. Lipid-associated changes were histologically consistent with human NAFLD. Liver tumors in diabetic males were consistent with HCC displaying cellular atypia and pleomorphism, local invasion into surrounding normal parenchyma, and a high mitotic index. HCCs were often the solid variant comprised primarily of clear cell hepatocytes. Mixed tumors with sublobules comprised eosinophilic cells in a trabecular or pelioid pattern were also represented (Fig. 9). Compared with diabetic males, no females $(P=0 \cdot 0079)$ or nondiabetic males $(P=0 \cdot 0667)$ developed liver tumors. Neither hepatitis nor fibrosis was a significant feature in any of the mice, and no significant hepatic lesions were observed in SW $<6$ months of age.

No significant cardiovascular or neurologic lesions were observed in these mice by the age of 12 months.

\section{Bacteriology}

Positive cultures obtained from urine were primarily mixed infections, and included Escherichia coli, Staphylococcus spp., Proteus spp., and Enterobacter aerogenes. Occasionally, Staphylococcus spp. were isolated from kidney, mandibular, and musculoskeletal abscesses.

Table 1 Kidney lesions in diabetic and nondiabetic colony SwissWebster (SW) mice at $\sim 6$ months

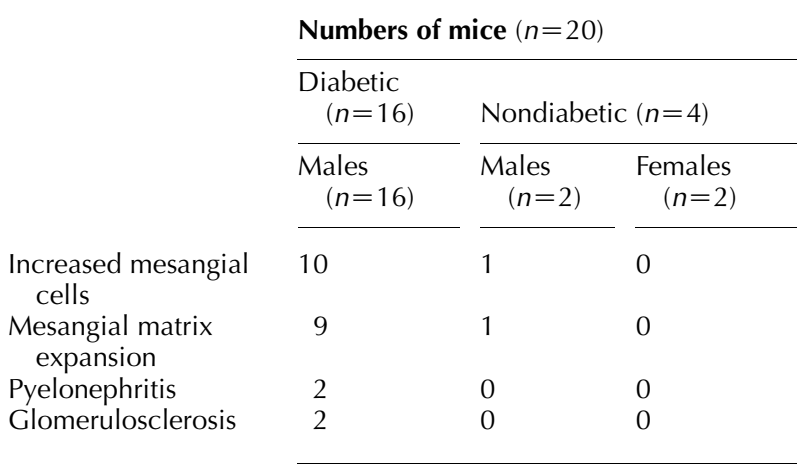

IHC

In diabetic male SW mice, degenerate islets showed patchy insulin positive cells and poor staining intensity compared with nondiabetic animals (Fig. 9). Islet cells positive for insulin were generally larger than surrounding cells and had prominent, vesicular nuclei, suggestive of compensatory hypertrophy. Insulin IHC confirmed the presence of islet metaplasia by demonstrating isolated insulin-producing cells within the exocrine pancreas and/or foci of insulinproducing cells admixed with exocrine-like cells in poorly defined islet-like sublobules as described previously (Fig. 9). Small nests of insulin-positive cells located adjacent to pancreatic tubules were suspected to represent islet neogenesis (Bouwens 2004), although specific cell lineage could not be determined morphologically. All female SW mice showed uniform, robust staining for insulin within islets. Isolated insulin-positive cells were extraordinarily rare in the exocrine pancreas of nondiabetic mice.

\section{Discussion}

We describe a cohort of outbred, male SW mice and their offspring with clinical and biochemical signs consistent with the metabolic syndrome and T2D mellitus in humans, characterized by insulin resistance and abdominal obesity. Clinical signs of T2D in male SW mice, including polyuria, polydipsia, and glucosuria were evident at an average age of 18 weeks. In these mice, a high incidence of diabetes was observed, approaching $60 \%$ in colony SW $(n=70)$ and $80 \%$ in dSWs $(n=20)$ by 30 weeks of age. The high prevalence of diabetes in the MIT SW breeding colony is suggestive of a founder effect - a loss of genetic variability initiated when the MIT colony was established from a relatively small number of animals from the original population of SW mice obtained from an outside source. Although mice from this colony are not typically assigned to research protocols, the effect of diabetes on the utility of SW mice for rodent health monitoring remains unknown. To limit the potential impact on rodent health, the MIT breeding colony was depopulated and renewed with additional mice from a different commercial vendor.

Initially, diabetic males showed increased body weight and grossly observable fat stores within the abdominal cavity compared with nondiabetic males; however, advanced disease resulted in declining body condition and loss of mesenteric and epididymal fat pads. Fasting glucose tolerance tests performed in diabetic mice (non-fasting blood glucose $>300 \mathrm{mg} / \mathrm{dl}$, glucosuria positive) confirmed altered glucose homeostasis compared with nondiabetic mice showing elevated glucose response curves with a delayed return to baseline. Glucose tolerance curves were variable between mice. This is likely a result of mice being in various stages of disease during glucose tolerance testing (10-13 months of age); however, concurrent illness such as liver tumors, pyelonephritis (bacterial), and/or 
dermatitis also resulted in variations in glucose measurement (mice with severe pyelonephritis frequently became euglycemic or slightly hypoglycemic). Curves were most consistent and predictable in known diabetic males, $\sim 10$ months of age with no appreciable concurrent illnesses. Although mice were at variable stages of disease during glucose tolerance testing (10-13 months of age), previously determined non-fasting hyperglycemia and glucosuria were predictive of elevated glucose response curves, despite the presence of liver tumors.

Diabetic colony SW and dSW males showed variable insulin levels that displayed a nonlinear correlation with blood glucose concentration. In the diabetic males (blood glucose $>300 \mathrm{mg} / \mathrm{dl}$ ), insulin levels were inversely proportional to the degree of hyperglycemia. Elevated serum insulin concentrations were seen at relatively mild stages of hyperglycemia. Mice with high insulin and mild hyperglycemia may reflect a compensated stage of diabetes with pancreatic $\beta$-cells producing increased insulin in response to insulin resistance. Increased $\beta$-cell mass has been described in numerous mouse models of spontaneous T2D, including the CBA/Ca mouse, and high-fat diet models (Orland \& Permutt 1987, Figueroa \& Taberner 1994, Sone \& Kagawa 2005), as well as humans (Bouwens \& Rooman 2005). In our outbred cohort, interindividual differences in pancreatic histopathologic presentation among diabetic males obscured any potential relationships between islet morphology and circulating insulin levels. $\beta$-cell degeneration and subsequent 'burnout' could result from glucotoxicity, lipotoxicity, or defective stimulation. This possibility is supported by the observation that $\beta$-cell degeneration was not seen in female mice. Importantly, $\beta$-cell degeneration with apoptosis and increasing levels of hyperglycemia have been described in diabetic rodents and humans, and are important in the pathogenesis of T2D (Asakawa et al. 2003, Butler et al. 2003, Medarova et al. 2005).

Although not directly correlated with circulating insulin or leptin levels, pancreata from male diabetic mice had mildto-severe islet cell degeneration with islet metaplasia. Regions of islet metaplasia were presumed to represent the transdifferentiation of pancreatic cells from an exocrine to an endocrine phenotype. Islet neogenesis, defined by poorly arranged islet cells in the normal periductular location, is reported in rodent models of pancreatic injury (streptozotocin administration, ischemia, subtotal pancreatectomy), transgenic mice, and in spontaneously hyperglycemic rats (Bouwens 2004, Topp et al. 2004, Peters et al. 2005). Additionally, neogenesis of human pancreatic islets has been described in obese and diabetic subjects (Butler 2003). However, to our knowledge, it has not been previously reported in spontaneous outbred mouse models of diabetes mellitus. Islet neogenesis is thought to occur as a compensatory mechanism to increase $\beta$-cell mass and number, due to increased insulin demands. The presence of histologic features such as the formation of mega-islets and transdifferentiation/neogenesis coupled with qualitatively inadequate production of insulin from these islets (patchy, sparse insulin staining by IHC compared with nondiabetic animals) is suggestive of a potential defect in islet production of insulin. However, the physiologic mechanisms underlying these compensatory responses are incompletely understood. Because this model shows a robust pancreatic islet cell response to the insulin resistant state, it should prove useful in exploring these mechanisms.

Similar to humans, the most notable features of the metabolic syndrome in the SW and $\mathrm{dSW}$ were abdominal obesity and insulin resistance. While a significantly higher lipemic index was observed in diabetic males compared with nondiabetic males, no grossly identifiable atherosclerotic plaques were seen in the proximal aorta, and systolic blood pressures were not measured. More extensive studies are required to fully ascertain the risk for CVD in this model. Unlike widely used mouse models of the metabolic syndrome, the $\mathrm{dSW}$ are maintained on a standard rodent diet, as opposed to experimental 'westernized' or high-fat diets. While environmental factors cannot be ignored, the $\mathrm{SW}$ and $\mathrm{dSW}$ may prove useful in exploring genetic mechanisms behind the development of the metabolic syndrome.

Diabetes mellitus, obesity, and hepatosteatosis are known risk factors for the development of HCC in human populations, predominantly in males (El-Serag 2004, Davila et al. 2005, Harrison 2006). Liver tumors consistent with clear cell HCC and mixed HCC were seen in $75 \%$ of aged, diabetic male SW and $\mathrm{dSW}(n=12)$ associated with a background macrosteatosis, microsteatosis, and hydropic change consistent with NAFLD in humans (Farrell \& Larter 2006). Studies of NAFLD in obese ob/ob mice have shown evidence of chronic liver injury with increased hepatocyte proliferation and decreased hepatocellular apoptosis that may explain an increased incidence of HCC in the ob/ob mice. However, like the $\mathrm{dSW}$ mice, ob/ob mice do not develop evidence of hepatitis or cirrhosis (Anstee \& Goldin 2006). Questions remain as to the role of hyperinsulinemia in carcinogenesis. High insulin levels, known hepatocyte mitogens, and growth promotants in murine and human HCC (Saito et al. 2002, Boissan et al. 2005) may have accelerated the growth of tumors resulting from unrelated causes or background genetic mutations. Although limited numbers of aged, nondiabetic male SW were available for study $(n=4)$, no gross tumors were seen in these mice or in aged nondiabetic females. dSW mice should provide a useful model to explore the mechanisms behind liver carcinogenesis associated with NAFLD in the diabetic state.

Additionally, in some aged mice with extensive HCC, a reversion to euglycemia or hypoglycemia was observed (data not shown). Hypoglycemia can occur in humans with HCC as a paraneoplastic syndrome associated with the production of insulin-like growth factors and/or through progressive malnutrition and increased glucose demands by neoplastic tissue (Yeung 1997). Generally, hypoglycemia associated with HCC is a poor prognostic indicator.

Selected diabetic male SW also showed histologic evidence of glomerulopathy independent of bacterial infection at less than 6 months of age, including basement membrane thickening and expansion of the mesangial matrix. This is 
similar to other mouse models of spontaneous diabetic nephropathy, including the $\mathrm{db} / \mathrm{db}$ mouse (Sharma et al. 2003); however, unlike the $\mathrm{db} / \mathrm{db}$ mouse, the $\mathrm{dSW}$ mice in this study lived beyond 12 months of age without food restriction and provide a useful model to study the natural progression of diabetic nephropathy. The dSW mice also revealed more severe diabetic kidney disease including membranous glomerulopathy as the animals aged. In these mice, the degree and progression of renal insufficiency, a feature lacking from most spontaneous mouse models of diabetes (Breyer et al. 2005) will require further studies.

$\mathrm{dSW}$ mice were also prone to bacterial infections of the urinary tract, in some cases resulting in severe renal disease. Mixed infections by opportunist bacteria were frequently cultured from kidney lesions and urine, including species of bacteria that are associated with urinary tract infection in human diabetics (Boyko et al. 2002) and in mouse models of T2D (Sone \& Kagawa 2005).

Although diabetic mice had moderately to markedly dilated gastrointestinal tracts, most notably in the cecum, no degenerative lesions were observed within small intestinal or cecal mesenteric plexi by light microscopy. However, electron microscopy and immunohistochemical staining may be required to demonstrate lesions of the autonomic nervous system consistent with diabetic neuropathy (Ordog et al. 2000). It is also possible that functional perturbances to intestinal motility unassociated with morphologic changes (e.g., to interstitial cells of Cajal) may play a role in the flaccid paralysis of the bowel that was a consistent gross feature of the diabetic males we analyzed. Similar gross lesions have been observed in diabetic rats (Karakida \& Homma 1989, Liu et al. 1990), proposed as a model of gastrointestinal autonomic neuropathy - a complication commonly seen in humans with type 1 diabetes (insulin dependent) mellitus. Signs of digestive dysfunction and delayed gastric emptying (gastroparesis) have also been described in human diabetics and mouse models of T2D (Asakawa et al. 2003, James et al. 2004). Gastroparesis without overt diabetes has also been noted by us in SW female mice (Garcia et al. 2001).

Clinical signs of T2D were not observed in female SW mice. Male predilection for diabetes is commonly reported in mouse models of spontaneous T2D, and may be due to the lack of protective effects of estrogen. In humans, prevalence and progression of diabetes increases in patients with conditions that result in decreased estrogen levels such as polycystic ovarian syndrome and menopause. Studies in humans and mice have also shown that estrogen replacement therapy improves glycemic control and decreases the incidence of diabetes (Louet et al. 2004).

Female SW mice displayed increased abdominal fat deposition by 10 weeks of age ( $>30 \mathrm{~g}$ body weight $(\mathrm{BW}))$ and marked obesity by 6 months ( $>50 \mathrm{~g} \mathrm{BW})$. While overt signs of diabetes were not observed, obese females occasionally developed gallstones without a cholesterol component (assessed by light microscopy utilizing a polarized light filter) that were consistent with either black or brown pigment stones. Gallstones are also seen with increased frequency in human diabetics and are thought to result from gallbladder hypomobility and decreased emptying associated with reduced cholecystokinin A receptor expression in biliary smooth muscle (Ding et al. 2005).

Although the female SW in our study consistently produced offspring between 2 and 4 months of age, no females became pregnant when bred after 8 months of age. This may be due to severe obesity rather than reproductive senescence. Dietary-induced obesity has been shown to reduce fertility by more than $60 \%$ in female DBA/2J mice with no effect on male fertility associated with hyperleptinemia and hypothalamic hypogonadism (Tortoriello et al. 2004). In the $\mathrm{db} / \mathrm{db}$ and ob/ob females, fertility is disrupted by mutation-induced hypercytolipidemic uteroovarian involution (Garris \& Garris 2004); however, histologic evidence of reproductive dysfunction was not found in the SW females. Also, no evidence of gestational diabetes was seen in pregnant SW females. The high successful pregnancy rate of the SW females less than 6 months of age, combined with their ability to successfully raise litters provides an advantage over infertile mouse models of T2D such as the $\mathrm{db} / \mathrm{db}$ mouse.

The dSW mice in this study share many features common to other mouse models of T2D including moderate obesity, maturity-onset glucosuria, and nephropathy. Importantly, additional relevant features include high reproductive performance, a strong pancreatic islet cell response to the diabetic state as evidenced by islet metaplasia, and relatively early-onset kidney disease with biochemical evidence of renal insufficiency, all of which make this model particularly useful (Table 2). Additionally, the reproductive ability of the female SW and variable alterations in the serum leptin concentrations of SW males suggest that the leptin/leptin receptor axis is not primarily involved in the pathogenesis of $\mathrm{T} 2 \mathrm{D}$ in this model similar to human T2D, and unlike the widely used $\mathrm{db} / \mathrm{db}$ and ob/ob mice. Other important characteristics include the SW mice's outbred background, and potentially the presence of autonomic neuropathy suggested by cecal and intestinal dilation, despite the lack of neuronal lesions visible by light microscopy. Importantly, the dSW spontaneously develops late-onset HCC, making them particularly useful for the study of diabetes-associated carcinogenesis.

Pathogenesis of diabetes in the SW follows a predictable course. At an early age, diabetic mice weigh significantly more than their nondiabetic counterparts and develop hyperglycemia at around 18 weeks of age. In mice less than 6 months of age, the pancreas attempts to compensate for disease by increasing the production of insulin, resulting in the presence of mega-islets, pancreatic transdifferentiation, and suspected islet neogenesis. As disease progresses, hyperinsulinemia is more prominent until the point of pancreatic decompensation, after which severe hyperglycemia may result $(>400 \mathrm{mg} / \mathrm{dl})$ as insulin levels decrease. At $\sim 6$ 
Table 2 Features of type 2 diabetes in selected spontaneous mouse models

\begin{tabular}{|c|c|c|c|c|}
\hline & Diabetic SW & $\mathrm{db} / \mathrm{db}$ & $\mathrm{KKA}^{\mathrm{y}}$ & NONcNZO10/Lt] \\
\hline \multicolumn{5}{|l|}{ Feature } \\
\hline Outbred & Yes & No & No & No \\
\hline Fertile & Yes & No & No & Yes \\
\hline Obesity & Moderate & Moderate-severe & Severe & Moderate \\
\hline Hyperglycemia & Moderate & Severe & Severe & Variable \\
\hline Leptin & Variable & Increased & Variable & Variable \\
\hline Nephropathy & Yes & Yes & Yes & ? \\
\hline Neuropathy & $?$ & Yes & Yes & $?$ \\
\hline Polygenic & $?$ & No & Yes & Yes \\
\hline
\end{tabular}

months of age, diabetic comorbidities such as kidney and liver diseases become apparent in diabetic males. By 12 months of age, most affected mice are entering end-stage disease with progressive cachexia, pancreatic disease, and liver disease (hepatosteatosis with hepatocellular degeneration and HCC). In end-stage diabetes, the pancreas may exhibit zymogen depletion and chronic exocrine pancreatitis due to suspected enzyme leakage from ectatic pancreatic ducts.

The genetic and physiologic mechanisms underlying the diabetic syndrome in this cohort of SW mice remain unknown and are the subjects of further investigation. SW mice retain the ability to breed, are normophagic, and have variable serum levels of leptin. In the dSW, serum leptin appears to be more dependent on body condition as diabetes progresses, blood glucose increases, fat stores are depleted, and serum leptin is also diminished. Other mechanisms may involve genes important in the transcription regulatory networks governing the liver and pancreatic islets such as the HNF transcription factors. Hnf1a, Hnf4a, and Onecut 1 are all required for the normal function of the liver and pancreas, and Hnf1a and Hnf4a mutations are known to cause maturity-onset diabetes of the young in humans (Odem et al. 2004). The dual involvement of the liver and pancreas in the dSW mice is suggestive of genetic defect affecting both liver and pancreas development; however, unlike some transgenic models of HNF dysfunction in mice that develop T2D and significant liver dysfunction early in life (Lee et al. 2003), the dSW mice develop liver pathology only after the development of late-stage diabetes. Microarray analyses are presently planned to examine the potential genetic contributions of various genes involved in liver and pancreas development to the pathogenesis of T2D and HCC; however, these studies may prove challenging to interpret due to the heterogeneity of the diabetic mice.

In conclusion, while this cohort of outbred SW male mice shares some physiologic and biochemical similarities with other mouse models of T2D, differences in parental background, reproductive capacity, and pancreatic response to disease make it a promising new model of the metabolic syndrome, T2D, and their comorbidities in humans. Further studies regarding the genetics and pathogenesis of the metabolic syndrome and T2D in this model are in progress.

\section{Declaration of interest}

The authors declare that there is no conflict of interest that could be perceived as prejudicing the impartiality of the research reported.

\section{Funding}

This work was supported by NIH grants P30 ES02 109 and T32 RR07036.

\section{Acknowledgements}

The authors gratefully acknowledge the work of Dr Sam Boutin and Heather Martin for their assistance in this study.

\section{References}

Accili D 1995 Molecular defects of the insulin receptor gene. Diabetes/ Metabolism Reviews 11 47-62.

Anstee QM \& Goldin RD 2006 Mouse models in non-alcoholic fatty liver disease and steatohepatitis research. International Journal of Experimental Pathology 87 1-16.

Asakawa A, Inui A, Ueno N, Makino S, Uemoto M, Fujino MA \& Kasuga M $2003 \mathrm{Ob} / \mathrm{ob}$ mice as a model of delayed gastric emptying. Journal of Diabetes and its Complications 17 27-28.

Beaver BV, Reed W, Leary S, McKiernan B, Bain F, Schultz R, Bennett BT, Pascoe P, Shull E \& Cork LC 20012000 Report of the AVMA Panel on Euthanasia. Journal of American Veterinary Medical Association 218 669-696.

Boissan M, Beurel E, Wendum D, Rey C, Lecluse Y, Housset C, Lacombe ML \& Desbois-Mouthon C 2005 Overexpression of insulin receptor substrate2 in human and murine hepatocellular carcinoma. American Journal of Pathology 167 869-877.

Bouwens L 2004 Islet morphogenesis and stem cell markers. Cell Biochemistry and Biophysics 40 81-88.

Bouwens L \& Rooman I 2005 Regulation of pancreatic beta-cell mass. Physiological Reviews 85 1255-1270.

Boyko EJ, Fihn SD, Scholes D, Chen CL, Normand EH \& Yarbro P 2002 Diabetes and the risk of acute urinary tract infection among postmenopausal women. Diabetes Care 25 1778-1783.

Breyer MD, Bottinger E, Brosius FC, Coffman TM, Fogo A, Harris RC, Heilig CW \& Sharma K 2005 Diabetic nephropathy: of mice and men. Advances in Chronic Kidney Disease 12 128-145.

Butler AE, Janson J, Bonner-Weir S, Ritzel R, Rizza RA \& Butler PC 2003 Beta-cell deficit and increased beta-cell apoptosis in humans with type 2 diabetes. Diabetes $\mathbf{5 2}$ 102-110.

Davila JA, Morgan RO, Shaib Y, McGlynn KA \& El-Serag HB 2005 Diabetes increases the risk of hepatocellular carcinoma in the United States: a population based case control study. Gut $\mathbf{5 4} 533-539$. 
Ding X, Lu CY, Mei Y, Liu CA \& Shi YJ 2005 Correlation between gene expression of CCK-A receptor and emptying dysfunction of the gallbladder in patients with gallstones and diabetes mellitus. Hepatobiliary and Pancreatic Diseases International 4 295-298.

El-Serag HB 2004 Hepatocellular carcinoma: recent trends in the United States. Gastroenterology 127 S27-S34.

Farrell GC \& Larter CZ 2006 Nonalcoholic fatty liver disease: from steatosis to cirrhosis. Hepatology 43 S99-S112.

Figueroa CD \& Taberner PV 1994 Pancreatic islet hypertrophy in spontaneous maturity onset obese-diabetic CBA/Ca mice. International Journal of Biochemistry 26 1299-1303.

Garcia A, Erdman S, Sheppard BJ, Murphy JC \& Fox JG 2001 Gastric dilatation syndrome associated with chronic nephropathy, hypergastrinemia, and gastritis in mice exposed to high levels of environmental antigens. Comparative Medicine 51 262-267.

Garris DR \& Garris BL 2004 Cytolipotoxicity-induced involution of the female reproductive tract following expression of obese (ob/ob) and diabetes $(\mathrm{db} / \mathrm{db})$ genotype mutations: progressive, hyperlipidemic transformation into adipocytic tissues. Reproductive Toxicology 18 81-91.

Gregg EW, Cadwell BL, Cheng YJ, Cowie CC, Williams DE, Geiss L, Engelgau MM \& Vinicor F 2004 Trends in the prevalence and ratio of diagnosed to undiagnosed diabetes according to obesity levels in the US. Diabetes Care 27 2806-2812.

Grundy SM, Brewer HB Jr, Cleeman JI, Smith SC Jr \& Lenfant C 2004 Definition of metabolic syndrome: Report of the National Heart, Lung, and Blood Institute/American Heart Association conference on scientific issues related to definition. Circulation 109 433-438.

Harrison SA 2006 Liver disease in patients with diabetes mellitus. Journal of Clinical Gastroenterology 40 68-76.

James AN, Ryan JP, Crowell MD \& Parkman HP 2004 Regional gastric contractility alterations in a diabetic gastroparesis mouse model: effects of cholinergic and serotoninergic stimulation. American Journal of Physiology. Gastrointestinal and Liver Physiology 287 G612-G619.

Karakida T \& Homma S 1989 Compliance changes of the gastrointestinal tract in streptozotocin-induced diabetic rats. Japanese Journal of Physiology 39 559-570.

Lee YH, Magnuson MA, Muppala V \& Chen SS 2003 Liver-specific reactivation of the inactivated $\mathrm{Hnf1}-\alpha$ gene: elimination of a liver dysfunction to establish a mouse MODY3 model. Molecular and Cellular Biology 23 923-932.

Liu HS, Karakida T \& Homma S 1990 In vitro motor activity and compliance of the caecum in streptozotocin diabetic rats. Japanese Journal of Physiology $\mathbf{4 0}$ 843-851.

Louet JF, LeMay C \& Mauvais-Jarvis F 2004 Antidiabetic actions of estrogen: insight from human and genetic mouse models. Current Atherosclerosis Reports 6 180-185.

McCarthy M \& Menzel S 2001 The genetics of type 2 diabetes. British Journal of Clinical Pharmacology 51 195-199.

Medarova Z, Bonner-Weir S, Lipes M \& Moore A 2005 Imaging beta-cell death with a near-infrared probe. Diabetes 54 1780-1788.

Odem DT, Zizlsperger N, Gordon DB, Bell GW, Rinaldi NJ, Murray HL, Volkert TL, Schreiber J, Rolfe PA, Giffor DK et al. 2004 Control of pancreas and liver gene expression by HNF transcription factors. Science 303 1378-1381.
Ordog T, Takayama I, Cheung WK, Ward SM \& Sanders KM 2000 Remodeling of networks of interstitial cells of Cajal in a murine model of diabetic gastroparesis. Diabetes 49 1731-1739.

Orland MJ \& Permutt MA 1987 Quantitative analysis of pancreatic proinsulin mRNA in genetically diabetic (db/db) mice. Diabetes 36 341-347.

Peters K, Panienka R, Li J, Kloppel G \& Wang R 2005 Expression of stem cell markers and transcription factors during the remodeling of the rat pancreas after duct ligation. Virchows Archiv 446 56-63.

Rees DA \& Alcolado JC 2005 Animal models of diabetes mellitus. Diabetic Medicine 22 359-370.

Riu E, Ferre T, Mas A, Hidalgo A, Franckhauser S \& Bosch F 2002 Overexpression of c-myc in diabetic mice restores altered expression of the transcription factor genes that regulate liver metabolism. Biochemical Journal 368 931-937.

Robertson DM \& Sima AA 1980 Diabetic neuropathy in the mutant mouse [C57BL $/ \mathrm{ks}(\mathrm{db} / \mathrm{db})]$ : a morphometric study. Diabetes 29 60-67.

Rogers AB, Taylor NS, Whary MT, Stefanich ED, Wang TC \& Fox JG 2005 Helicobacter pylori but not high salt induces gastric intraepithelial neoplasia in B6129 mice. Cancer Research 65 10709-10715.

Rosenbaum M, Nonas C, Horlick M, Fennoy I, Vargas I, Schachner H, Kringas P, Stanton K \& Weil R 2004 Beta-cell function and insulin sensitivity in early adolescence: association with body fatness and family history of type 2 diabetes mellitus. Journal of Clinical Endocrinology and Metabolism 89 5469-5476.

Saito K, Inoue S, Saito T, Kiso S, Ito N, Tamura S, Watanabe H, Takeda H, Misawa H, Togashi H et al. 2002 Augmentation effect of postprandial hyperinsulinaemia on growth of human hepatocellular carcinoma. Gut $\mathbf{5 1}$ 100-104.

Sharma K, McCue P \& Dunn SR 2003 Diabetic kidney disease in the $\mathrm{db} / \mathrm{db}$ mouse. American Journal of Physiology. Renal Physiology 284 F1138-F1144.

Sone H \& Kagawa Y 2005 Pancreatic beta cell senescence contributes to the pathogenesis of type 2 diabetes in high-fat diet-induced diabetic mice. Diabetologia 48 58-67.

Sullivan PW, Morrato EH, Ghushchyan V, Wyatt HR \& Hill JO 2005 Obesity, inactivity, and the prevalence of diabetes and diabetes-related cardiovascular comorbidities in the US, 2000-2002. Diabetes Care 28 1599-1603.

Topp BG, McArthur MD \& Finegood DT 2004 Metabolic adaptations to chronic glucose infusion in rats. Diabetologia 47 1602-1610.

Tortoriello DV, McMinn J \& Chua SC 2004 Dietary-induced obesity and hypothalamic infertility in female DBA/2J mice. Endocrinology 145 $1238-1247$.

Ullman-Cullere MH \& Foltz CJ 1999 Body condition scoring: a rapid and accurate method for assessing health status in mice. Laboratory Animal Science 49 319-323.

Ye G, Donthi RV, Metreveli NS \& Epstein PN 2005 Cardiomyocyte dysfunction in models of type 1 and type 2 diabetes. Cardiovascular Toxicology 5 285-292.

Yeung RTT 1997 Hypoglycaemia in hepatocellular carcinoma: a review. Hong Kong Medical Journal 3 297-301.

Received in final form 21 July 2008

Accepted 29 July 2008

Made available online as an Accepted Preprint 30 July 2008 